\title{
Clinico-
pathological
conference
}

\section{Haemolytic uraemic syndrome complicated by disseminated extraneural cryptococcosis}

\author{
A L Pozniak, S B Lucas, R F Miller
}

\section{Case report (Dr R F Miller)}

A 33 year unemployed white man was admitted to hospital in January 1994 complaining of a 1 week history of diarrhoea, passing up to 30 stools per day. The stool consisted of watery yellow fluid without blood or mucus. He reported associated anorexia with intermittent nausea and vomiting together with left lower quadrant colicky abdominal pain and sweats but no fever. The patient had been seen in a local casualty department 4 days previously at which time he had reported 3 days of diarrhoea. At that time he reported that he was HIV antibody positive and examination had revealed no abnormalities. Investigations revealed normal urea and electrolytes, a haemoglobin of $10.4 \mathrm{~g} / \mathrm{dl}$, a total white blood count of $6.8 \times 10^{9} / 1$, and a platelet count of $68 \times 10^{9} / 1$. This latter finding was ascribed to the effects of HIV infection. The patient was treated symptomatically and discharged.

On admission to hospital in January 1994 the patient reported that 72 months previously he had developed night sweats, gingivitis, and oral candidiasis. He had been admitted to another hospital in April 1988 with a chest infection and, in May of that year following a party, he had been admitted to the Middlesex Hospital with diarrhoea and vomiting. He subsequently reported that he had passed malaena stools and, in addition, stated that he had been found to be HIV positive in October 1987; this positive result was performed on a sample of serum stored from 1982. The investigation had been carried out at another hospital. On that admission in 1988 examination (including rectal examination) was unremarkable. Sigmoidoscopy and biopsy were normal and the patient was noted to have an inappropriate effect with a rather dramatic story. Investigations revealed a normal full blood count. During the admission the patient was counselled and underwent HIV testing: the results were positive. The diagnosis at that time was one of Munchausen's/malingering.

Subsequently, the patient had experienced chronic diarrhoea and had attended many different clinics. In the year before his admission with diarrhoea Cryptosporidium parvum had been isolated repeatedly in the stool. Six months before admission the patient had experienced recurrent episodes of perianal herpes simplex virus infection and began aciclovir suppression. Two months before admis- sion his CD4 count had fallen from $290 \times$ $10^{6} / 1$ to $110 \times 10^{6} / 1$. The patient had declined antiretroviral therapy but had begun co-trimoxazole as primary prophylaxis against Pneumocystis carinii pneumonia. At this stage the patient also reported that in 1987 he had cytomegalovirus (CMV) colitis and hepatitis which were treated with foscarnet. This episode had occurred at another hospital. Contact with that hospital revealed no patient of that name. On further inquiry the patient reported a family history of haemophilia, he was unable to say of what sort. His social history revealed that he was unemployed but had worked in several different jobs. His homosexual life style was his risk factor for HIV infection. He had no travel history of note.

On examination in January 1994 he was pyrexial with a temperature of $38 \cdot 2^{\circ} \mathrm{C}$. He had shotty lymphadenopathy in the right inguinal region. In the abdomen there was generalised tenderness but no organomegaly or masses: rectal examination was unremarkable. The rest of the examination was normal; he was normotensive. Initial investigations included a CMV DEAFF test which was positive in urine and negative in blood. Culture of urine, blood, and stool were negative for bacteria, fungi, protozoa, and acid and alcohol fast bacilli (AAFB). Sigmoidoscopy revealed a mildly inflamed mucosa but biopsy was normal. Urea and electrolytes were repeated. The patient refused to stay in hospital but returned to the ward the following day having sustained a soft tissue injury to his right thumb. This had happened as he had fallen at home. Having been seen and assessed he left the ward once more and again returned the following day; at that time he was verbally aggressive to nursing and medical staff. He made a self diagnosis of HIV myelopathy and he based this diagnosis on the fact that he was unable to walk downstairs. $\mathrm{He}$ was noted to have uncontrollable spasms of his arms and legs but declined investigations of these symptoms. Four days after his original admission he once more returned to the ward at which time he was apyrexial and his diarrhoea had ceased. On re-examination the abdomen was still diffusely mildly tender and the rest of the examination was normal. In particular there were no neurological abnormalities, no rash, and no signs of spontaneous bleeding. Investigations revealed that the haemoglobin was now $6.5 \mathrm{~g} / \mathrm{dl}$, the platelet count had fallen to $74 \times 10^{9} / 1$, and the serum 
creatinine had risen to $416 \mu \mathrm{mol} / \mathrm{l}$. A peripheral blood film showed many red cell fragments; a clotting screen showed prothrombin time was 14/12 seconds and thrombin time was $1 \cdot 24$. A blood film for malaria parasites was negative. A Coombs test was negative and haptoglobin levels were reduced. Urinanalysis showed blood +++ and protein +++ ; urine microscopy revealed casts and red blood cells. An EEG was done because of the spasms and this was normal. A provisional diagnosis lay between a thrombotic microangiopathy such as haemolytic uraemic syndrome or disseminated intravascular coagulopathy.

The patient was managed with resalination, intravenous fresh frozen plasma, whole blood, and intravenous broad spectrum antibiotics. Over the next 72 hours he remained clinically stable and received further blood transfusions and intravenous fresh frozen plasma. He remained normotensive. Seven days after his original admission to the ward he became acutely unwell with pyrexia up to $40^{\circ} \mathrm{C}$, tachypnoeic at 30 breaths per minute, and had a sudden recurrence of diarrhoea. Examination revealed diffuse abdominal tenderness but was otherwise unremarkable. Investigations showed that the $\mathrm{PaO}_{2}$ (taken with the patient breathing room air) = $5.9 \mathrm{kPa}$, and the $\mathrm{PaCO}_{2}=3.0 \mathrm{kPa}$. Legionella and mycoplasma serology were negative. A chest radiograph was unremarkable. It was felt that $P$ carinii pneumonia was a possible diagnosis and so intravenous high dose co-trimoxazole was begun as empirical therapy together with adjuvant methylprednisolone. Intravenous erythromycin was also given to treat a possible atypical pneumonia. Supplemental oxygen was given by face mask. Review later that day showed that the $\mathrm{PaO}_{2}$ (breathing 60\% oxygen via face mask) $=12.7 \mathrm{kPa}$. The patient felt clinically better; physical signs revealed evidence of fluid overload only. The following day (day 9 of the admission) it was noted that urine output was poor despite frusemide. The jugular venous pressure was $+3 \mathrm{~cm} \mathrm{H}_{2} \mathrm{O}$, blood pressure $=130 / 90$. Fresh frozen plasma and blood were transfused. The patient remained hypoxaemic, while breathing room air, $\mathrm{SaO}_{2}=85 \%$. A repeat chest radiograph showed diffuse interstitial infiltrates. It was felt that bronchoscopy was too risky because of the low oxygen saturation, the low platelet count, and the abnormal renal function. At this stage the creatinine had risen to $512 \mu \mathrm{mol} / 1$ and the urea to $37 \cdot 8 \mathrm{mmol} / \mathrm{l}$. The haemoglobin, despite blood transfusion, remained low at $8.3 \mathrm{~g} / \mathrm{dl}$. Intravenous prostacycline was added to the therapeutic regimen at this point. The patient's condition steadily deteriorated with marked fluid retention, oedema of the sacrum and ankles, and crackles at both lung bases. A chest radiograph taken on day 13 was markedly abnormal, showing diffuse interstitial and alveolar infiltrates (fig 1). The $\mathrm{PaO}_{2}$ (breathing $60 \%$ oxygen via a face mask) $=6.8 \mathrm{kPa}$. The patient was transferred to the intensive care unit for continuous positive airway pressure (CPAP) ventilation and haemofiltration. Mask CPAP was temporarily

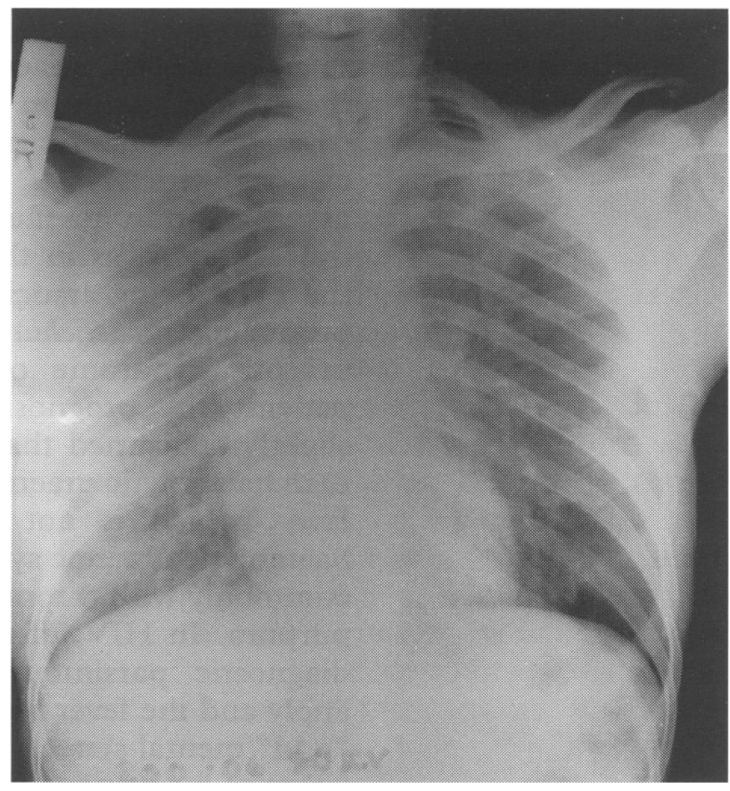

Figure 1 Chest radiograph, taken on day 13 of admission, showing diffuse interstitial and alveolar infiltrates.

successful but was confounded by increasing positive end expiratory pressure requirements in order to maintain oxygen saturations. At this point the patient refused ongoing treatment. After discussions between the medical staff, the patient, and his family and friends he was transferred back to a general ward where he died shortly afterwards. An investigation performed shortly before death subsequently revealed the diagnosis.

\section{Discussion (Dr A L Pozniak)}

To summarise, this HIV infected 33 year white man with a CD4 count of $110 \times 10^{6 / 1}$ presented with acute on chronic diarrhoea, fever, and abdominal pain. He was found to be in renal failure and to have anaemia and thrombocytopenia, the peripheral blood film showed red blood cell fragments. $\mathrm{He}$ was treated for haemolytic uraemic syndrome and, with this as the background, developed a fulmininant pneumonia and died. An investigation performed just before death revealed the diagnosis.

I would like to begin by focusing on this patient's haematological problem. The differential diagnosis lies between disseminated intravascular coagulopathy or a thrombotic microangiopathy. Disseminated intravascular coagulopathy can be excluded because of the normal clotting screen. The thrombotic microangiopathies are a closely related group of clinical syndromes which include thrombotic thrombocytopenic purpura and haemolytic uraemic syndrome. They are characterised by microangiopathic haemolytic anaemia, thrombocytopenia, intravascular thrombosis, and end organ failure. ${ }^{1}$ The kidney is the organ predominantly affected by haemolytic uraemic syndrome whereas cerebral dysfunction is a major feature of thrombotic thrombocytopenic purpura-thus, distinction between the two syndromes may often be made on clinical grounds. 
Thrombotic thrombocytopenic purpura rather than haemolytic uraemic syndrome has been the clinical picture in the majority of reported cases of thrombotic microangiopathy in association with $\mathrm{HIV}^{2}$ Onset of haemolytic uraemic syndrome may be preceded by a diarrhoeal illness, as in this case, the majority of the cases being associated with verocytotoxin producing Escherichia coli. ${ }^{3}$ When haemolytic uraemic syndrome occurs in HIV infected patients the prognosis is very poor. ${ }^{1}$ I am slightly concerned that this patient had more than haemolytic uraemic syndrome as he had a fever, which is not a common feature of haemolytic uraemic syndrome-it occurs more commonly with thrombotic thrombocytopenic purpura. In HIV infection Occam's razor (of diagnostic parsimony) frequently does not apply and the fever together with the apparent "odd" mental state, which may have been due either to his personality or to hypoxia or possibly CNS disease, make me wonder whether there was a second disease process going on.

In order to complete the diagnostic evaluation there are several pieces of additional information and results of investigations that I would have liked to have known. I note that there was no focal neurological abnormality on examination but I wonder, in view of the history of spasms of his limbs, what a computed tomographic or magnetic resonance scan of the head would have shown? Also analysis of cerebrospinal fluid, at lumbar puncture, might have shown a chronic meningitis or evidence of herpesvirus infection; even a negative result would have been reassuring. You did not do a lumbar puncture, firstly, because the "flavour" of the original presentation was nephrological, and, secondly, because of the abnormally low platelet count and abnormal platelet function in uraemia which would have made it a hazardous procedure to perform.

The finding of a normal chest radiograph in a hypoxic patient raises several possibilities. The most likely explanation, as in this case, is that of a pneumonia in evolution. The following chest radiograph only a few days later was grossly abnormal. An alternative diagnosis that needs to be considered is pulmonary embolism which is unlikely given the haematological abnormalities. Fluid overload could have been the cause; however, you monitored fluid replacement with a central venous pressure line and also the patient never became hypertensive. Acute bacterial pneumonia or septicaemia and adult respiratory distress syndrome (ARDS) have to be considered, but the patient was given broad spectrum antibiotics and was not neutropenic. The cause is unlikely to have been adenovirus or legionella pneumonia given the time course. However, the negative "acute" serology does not exclude a diagnosis of legionella pneumonia.

Cryptococcal pulmonary infection in the context of AIDS usually produces nodular abnormalities, with or without lymphadenopathy, on the chest radiograph. ${ }^{45}$ However, diffuse interstitial shadowing or alveolar consolidation, or both, may occur-mimicking $P$ carinii pneumonia. ${ }^{6}$ In common with many other infections, including $P$ carinii pneumonia, ${ }^{7}$ pulmonary cryptococcal infection may produce ARDS with "white out" on the chest radiograph. ${ }^{8}$ It would have been useful to have a cryptococcal latex agglutination (CRAG) titre from peripheral blood in this case.

I think that there are several decoys in this case. Firstly, Cryptosporidium parvum was isolated repeatedly in this man's diarrhoea stool. $C$ parvum may colonise epithelial surfaces including the trachea and the lungs, rarely it may produce respiratory symptoms. The outcome when it does even without treatment is good, in contrast to this case. ${ }^{9}$ Secondly, CMV was detected in urine by DEAFF. This is a very common finding in homosexual HIV infected men and is due to reactivation of virus and does not always imply active disease. The retinal appearances in this case were normalthere were no changes of CMV retinitis. The finding of CMV in the lungs of patients with AIDS is common, but the virus is simply a passenger requiring no specific treatment and co-exists with other pathology. ${ }^{10}$ In contrast, in HIV infected patients with higher than average CD4 lymphocyte counts CMV may cause a severe pneumonitis which is immunopathologically mediated. ${ }^{11}$ The patient was considered too sick to undergo fibreoptic bronchoscopy and alveolar lavage. This procedure may well have been diagnostic. This man's personality and his previous history of feigned illness are also peripheral to the underlying problems of haemolytic uraemic syndrome and pneumonia. Feigned HIV infection, malingering, and Munchausen's syndrome have all been described previously. ${ }^{1213}$

If one ties together the pulmonary and neurological problems and looks for a unifying diagnosis then conditions such as toxoplasmosis, lymphoma, and tuberculosis come to mind. It would be unusual for an HIV infected patient with tuberculosis meningitis to also have an acute tuberculous pneumonia. ${ }^{14}$ Disseminated cryptococcal infection is something that needs to be seriously considered-it could account for the neurological features, if a meningitis was present, and systemic infection might account for the fever and fulminant pneumonia. We are told that a sample obtained immediately before death gave diagnostic information. Was this a blood culture result, showing perhaps a mycobacterial or fungal species? Was it a result from urine or stool? Given the patient had a respiratory illness as his agonal event could the sample have been sputum? Mycobacteria and also fungi, including Aspergillus and Cryptococcus neoformans, may be diagnosed by conventional staining and culture of spontaneously expectorated sputum and $P$ carinii may be diagnosed by the polymerase chain reaction applied to sputum or mouthwash. ${ }^{15}$ Was it perhaps a serological diagnosis, either a rise in specific antibodies, ํㅡㄹ for example, to mycoplasma or legionella, or was it a positive CRAG result?

In summary I think this patient had haemolytic uraemic syndrome, ARDS, and another underlying disease process, probably an opportunistic infection. I am uncertain 
whether this was fungal-for example, $C$ neoformans, Histoplasma capsulatum, or mycobacterial-for example, Mycobacterium tuberculosis. I think the sample obtained just before death was probably sputum.

\section{CLINICAL DIAGNOSES}

(1) Haemolytic uraemic syndrome

(2) Adult respiratory distress syndrome

(3) An opportunistic infection-fungal? -mycobacterial?

\section{Necropsy findings (Professor S B Lucas)}

Before addressing the necropsy findings, I reviewed the several rectal biopsies taken from 1988 onwards. The 1988 sample was not normal, but showed intestinal spirochaetosis. That from 1994 was normal, despite continued diarrhoea and the subsequent finding of cryptosporidiosis in the small bowel at necropsy.

The cadaver had pallor and pitting oedema up to the knees, but was not emaciated. The tongue and oesophagus showed candidiasis. The major macroscopic pathologies were in the lungs, kidneys, liver, spleen, and lymph nodes. The lungs' combined weight was $3700 \mathrm{~g}$ (normal about $500 \mathrm{~g}$ ) and showed a uniform dark red consolidation (indicating shock lung). The kidneys were enlarged (combined weight $605 \mathrm{~g}$; normal about $300 \mathrm{~g}$ ), with blotchy oedematous cortices suggesting actue tubular necrosis. Both liver ( $3000 \mathrm{~g}$; normal $1500 \mathrm{~g}$ ) and spleen (530 g; normal $200 \mathrm{~g}$ ) were grossly enlarged and pale, this is a macroscopic clue to aetiology since the main causes of such an appearance are Mycobacterium avium complex or cryptococcal infections. The heart was large at $450 \mathrm{~g}$ (normal $300 \mathrm{~g}$ ) but without focal lesions; the brain (1770 g), spinal cord, and eyes were macroscopically normal.

The histology samples showed two major pathologies. There was massive infection by Cryptococcus neoformans, involving the lungs (figs 2 and 3), liver, spleen, bone marrow, and lymph nodes. Also infected were the heart, adrenal glands, pancreas, kidneys, and choroid of the eye.

The brain and spinal cord meninges and parenchyma were not involved, and nor was there HIV or CMV encephalitis. Secondly, the kidneys, in addition to interstitial and glomerular cryptococcosis, showed the features of haemolytic uraemic syndrome: fibrin thrombi and fibrinoid necrosis in glomeruli and afferent arterioles, glomerular collapse and, with silver stains, double contours in glomerular loops, and acute tubular necrosis (fig 4).

Additional pathologies were CMV infection of the adrenal and cryptosporidiosis of the ileum. The lungs also had hyaline membrane disease (shock lung), but there was no $P$ carinii infection or mycobacterial infection.

In summary, this is a classic case where the necropsy histopathology proved definitive; the gross appearances were, as so often in HIV/AIDS, non-specific. The patient died of haemolytic uraemic syndrome and cryptococal

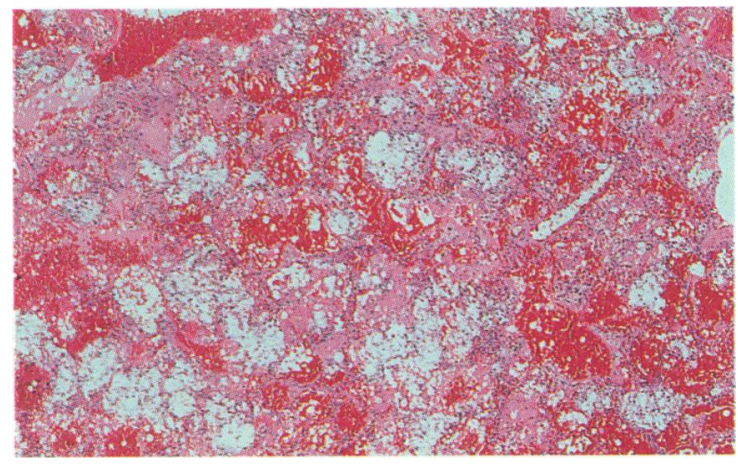

Figure 2 Lung showing cryptococcal pneumonia. The pale aggregates are clusters of fungi amid shock lung. Haematoxylin and eosin. Low power.

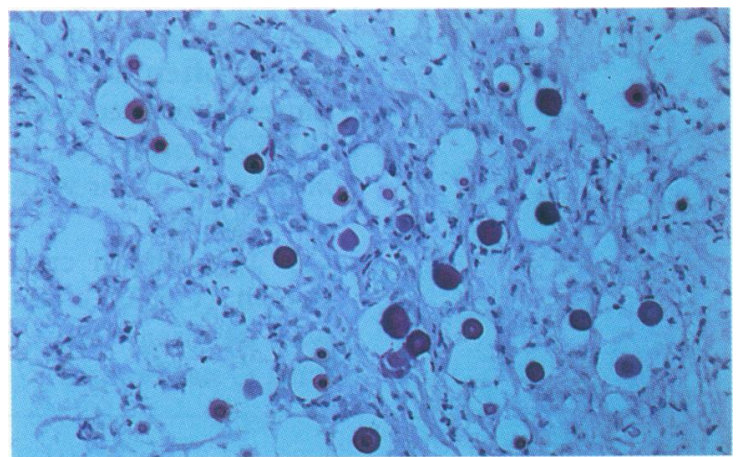

Figure 3 Lung showing cryptoccal pneumonia. There are magenta staining yeasts with surrounding pale (nonstaining) capsular mucoid material. Periodic acid Schiff. High power.

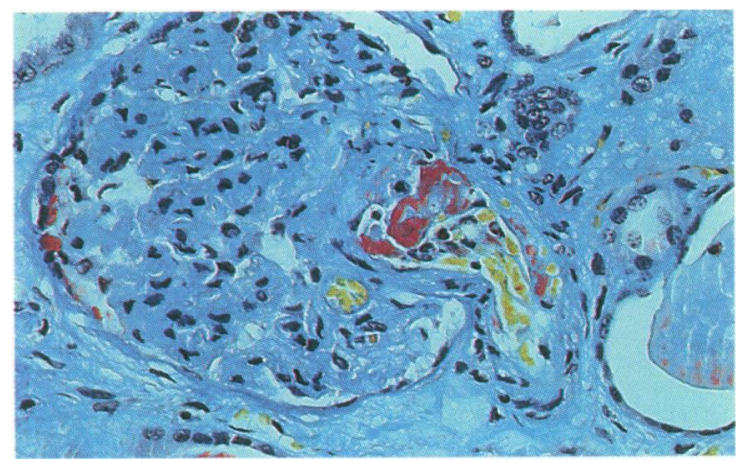

Figure 4 Renal glomerulus showing fibrin within the afferent arteriole (red staining) and collapse of the glomerular segments. Trichrome. High power.

pneumonia. This patient had visceral but not cerebral cryptococcosis. This is not unusual. One necropsy series in west Africa found a 3\% prevalence of cryptococcosis among unselected HIV positive adult deaths on medical wards; all had pulmonary disease but only three quarters had cerebral involvement. ${ }^{16} \mathrm{~A}$ similar distribution is recorded in series from North America, in one 89/106 (84\%) of HIV infected patients with $C$ neoformans infection had CNS involvement, ${ }^{17}$ in another 51/63 $(81 \%)$ had CNS disease. ${ }^{18}$

\section{PATHOLOGICAL DIAGNOSES}

Major pathology

(1) Haemolytic uraemic syndrome

(2) Disseminated extraneural cryptococcal infection

(3) Hyaline membrane disease (shock lung)

Minor pathology

(4) CMV adrenalitis

(5) Cryptosporidiosis of the ileum 


\section{Dr R F Miller}

The sample obtained just before death was sputum which contained $C$ neoformans. With hindsight we should have performed a serum CRAG test. This assay detects cryptococcal polysaccharide capsular antigens using antibody coated particles ${ }^{19}$ and is usually positive in serum of patients with both cryptococcal meningitis and disseminated extraneural cryptococcosis.

\section{Key points}

(1) Haemolytic uraemic syndrome is an unusual cause of thrombotic microangiopathy in HIV infection: it has a poor prognosis. (2) Occam's razor (of diagnostic parsimony) often does not apply in HIV infected patients with advanced disease.

(3) Disseminated infection with $C$ neoformans may occur without CNS or meningeal involvement.

(4) The serum cryptococcal latex agglutination (CRAG) test may provide a rapid way of diagnosing disseminated infection with $C$ neoformans.

\section{Footnote}

This case was presented as an unseen case at the Medical Staff Grand Round at University College London Hospitals on 26 February 1997. It was presented again at the meeting of the MSSVD on 25 April 1997, when Dr B G Gazzard was the discussant.

We thank Louise Hinds for typing the manuscript.

1 Kelleher R, Severn A, Tomson C, Lucas S, Parkin J, Pinching A, et al. The haemolytic uraemic syndrome in patients with AIDS. Genitourin Med 1996;72:172-5.

2 Leaf AN, Laubenstein LJ, Raphael B. Thrombotic thrombocytopenia purpura associated with human immuno- deficiency virus type-1 (HIV) infection. Ann Intern Med 1988;109:194-7.

3 Boyce TG, Swerdlow DL, Griffin PM. Current concepts Escherichia coli $0157: 7 \mathrm{H}$ and the hemolytic uremic syndrome. $N$ Engl $\mathcal{F}$ Med 1995;333:364-8.

4 Miller WT, Edelman JM, Miller WT. Cryptococcal pulmonary infection in patients with AIDS: radiographic appearance. Radiology 1990;175:725-8.

5 Clark RA, Greer DL, Valainis GT, Hyslop NE. Cryptococcus neoformans pulmonary infection in HIV-1 infected coccus neoformans pulmonary infection in HIV-1
patients. f Acq Imm Defic Syndr 1990;3:480-4.

6 Batungwanayo J, Taelman H, Bogaerts J, Allen S, Lucas S, Kagame A, et al. Pulmonary cryptococcosis associated with HIV-1 infection in Rwanda: a retrospective study of 37 cases. AIDS 1994;8:1271-6.

7 Scoular A, Moxham J, Lucas SB, Miller RF. Adult respiratory distress syndrome complicating Pneumocystis carini pneumonia. Genitourin Med 1991;67:250-5.

8 Murray RJ, Becker P, Firth P, Criner GJ. Recovery from cryptococcaemia and the adult respiratory distress syndrome in the acquired immunodeficiency syndrome. Chest 1988;93:1304-6.

9 Hojlyug N, Nybo Jensen B. Respiratory cryptosporidiosis in HIV-positive patients. Lancet 1988;i:590-1.

10 Millar AB, Patou G, Miller RF, Grundy JE, Katz DR, Weller IV, et al. Cytomegalovirus in the lungs of patients with AIDS. Am Rev Respir Dis 1990;141:1474-7.

11 Squire SB, Lipman MG, Bagdades EK, Mulvenna PM, Grundy JE, Griffin PD, et al. Severe cytomegalovirus pneumonitis in HIV infected patients with higher than average CD4 counts. Thorax 1992;47:301-4.

12 Churchill DR, De Cock KM, Miller RF. Feigned HIV infection/AIDS: malingering and Munchausen's syndrome. Genitourin Med 1994;70:314-6.

13 Parmar M, Boag F, Jayasuriya P, Catalan J. Feigned HIV disease. Int 7 STD AIDS 1990;1:447.

14 Dickens P. Fulminant tuberculous bronchopneumonia in a young Hong Kong Chinese woman. Pathology 1991;23: 249-9.

15 Wakefield AK, Miller RF, Guiver LA, Hopkin JM. Oropharyngeal samples for detection of Pneumocystis carinii by DNA amplification. $Q \mathcal{F} M e d$ 1993;86:401-6.

16 Lucas SB, Hounnou A, Peacock C, Beaumel A, Djomand G, N'Gbichi J-M, et al. The mortality and pathology of HIV infection in a West African city. AIDS 1993;7: 1569-80.

17 Chuck SL, Sande MA. Infections with cryptococcus neoformans in AIDS. $N$ Engl 7 Med 1989;321:794-9.

18 Clark RA, Greer D, Atkinson W. Spectrum of Cryptococcus neoformans infection in 68 patients infected with HTV. neoformans infection in 68 patien

19 Powderly WG, Cloud GA, Dismukes WE, Saag MS Measurement of cryptococcal antigen in serum and cerebrospinal fluid: value in the management of AIDS-associated cryptococcal meningitis. Clin Infect Dis 1994;18 789-92. 